\title{
Applications of Focus Ion Beam Technique in the Characterization of Nanocrystal Nonvolatile Memory Devices
}

\author{
Jian-Guo Zheng
}

The Laboratory for Electron and X-ray Instrumentation, California Institute for Telecommunication and Information Technology (Calit2), University of California, Irvine, CA 92697-2800

Nanocrystal nonvolatile memory devices have attracted considerable attention due to their variety of advantages, such as high-density integration and low power consumption. The nanocrystals and some fine key com ponents in the devices are cl osely related to device performance and their microstructures need to be carefully characterized and controlled. Transmission electron microscopy (TEM) is a commonly used characterization tool, however, a successful TEM experiment is strongly dependent on specim en preparation. Therefore, it is ver y important to prepare a site-sp ecific electron-transparent thin specimen for TEM observation so that the observed microstructures can be correlated to device performance.

Focus ion beam in a dual-beam system is succe ssfully applied to pr epare such kinds of TEM specimens. Nanocrystal nonvolatile $m$ emory devices st udied in this paper include two categories, that is, nanocrystal floating gate $\mathrm{m}$ emory and resistive random access memory. Fig. 1a shows the cross-sectional TEM sp ecimen of NiSi nanocrystal floating gate memory, prepared by focus ion beam (FIB) technique. The key device com ponents consisting of NiSi nanocrystals embedded between $\mathrm{SiO}_{2}$ tunnel layer and control layer can be clearly seen. Besides crystal size and shape, this TEM specimen allows us to obtain high resolution im ages of the nanocrystals down to atom ic scale (Fig. 1a inset). In addition, plane-view specim ens from a selected memory unite can be prepared as well (Fig. 1b), providing supplem ental information about the density a nd uniformity of these nanocrystals sandwiched between $\mathrm{Al}$ electrodes. $\mathrm{ZnO}$ nanocystals grown on Si substrate are used for resistive random access memory. The TEM specim ens were successfully prepared by FIB as well (Fig. 2a), which enable us to understand the microstructure of $\mathrm{ZnO}$ nanocrystals and the relationship between the nanocrystals and the substrate (Fig. 2b).

During TEM specim en preparation by m eans of FIB, some methods were a pplied to effectively protect the nanocrystals from ion beam induced damage, avoid the contam ination of Pt particles in the nanocrystal area, and minimize charging problems resulted from the non-conducting oxide layers.

In conclusion, FIB is a unique and convenient techni que to prepare site-specific electron-transparent thin TEM specim ens. This is especially true for characterizing nanocr ystal nonvolatile m emory devices, where the device thickness is only a couple of hundred nanometers.

[1] The samples are provided by H.M Zhou, J.J. Ren and J. Qi from Prof. Jianlin Liu's group. 

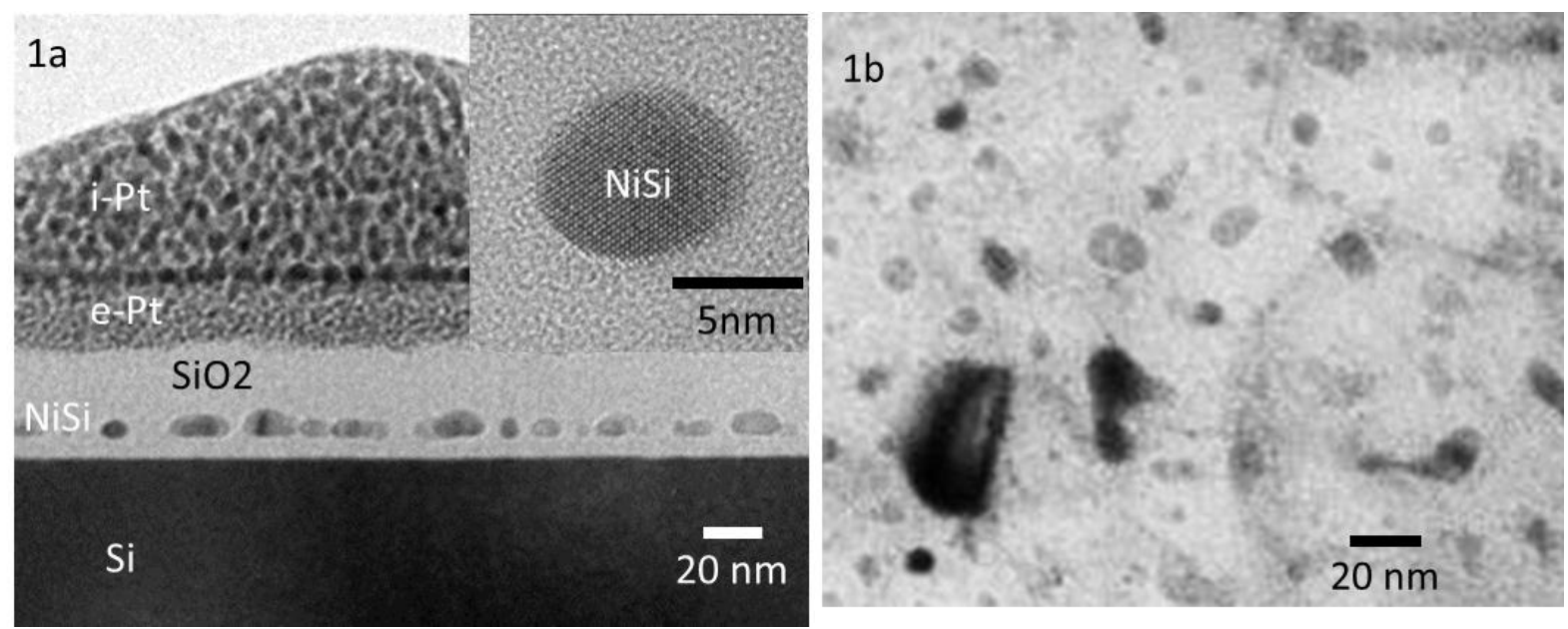

Fig. (1a) A cross-sectional TEM specimen of NiSi nanocrystal floating gate memory prepared by FIB technique and imaged by a TEM. The inset shows the high resolution lattice image of a NiSi crystal on an atomic scale. (1b) Plane-view TEM specimens from a selected memory unite, providing information about the density and uniformity of these nanocrystals sandwiched between electrodes. Please note that the big black grain is related to Al electrode instead of NiSi nanocrystals.
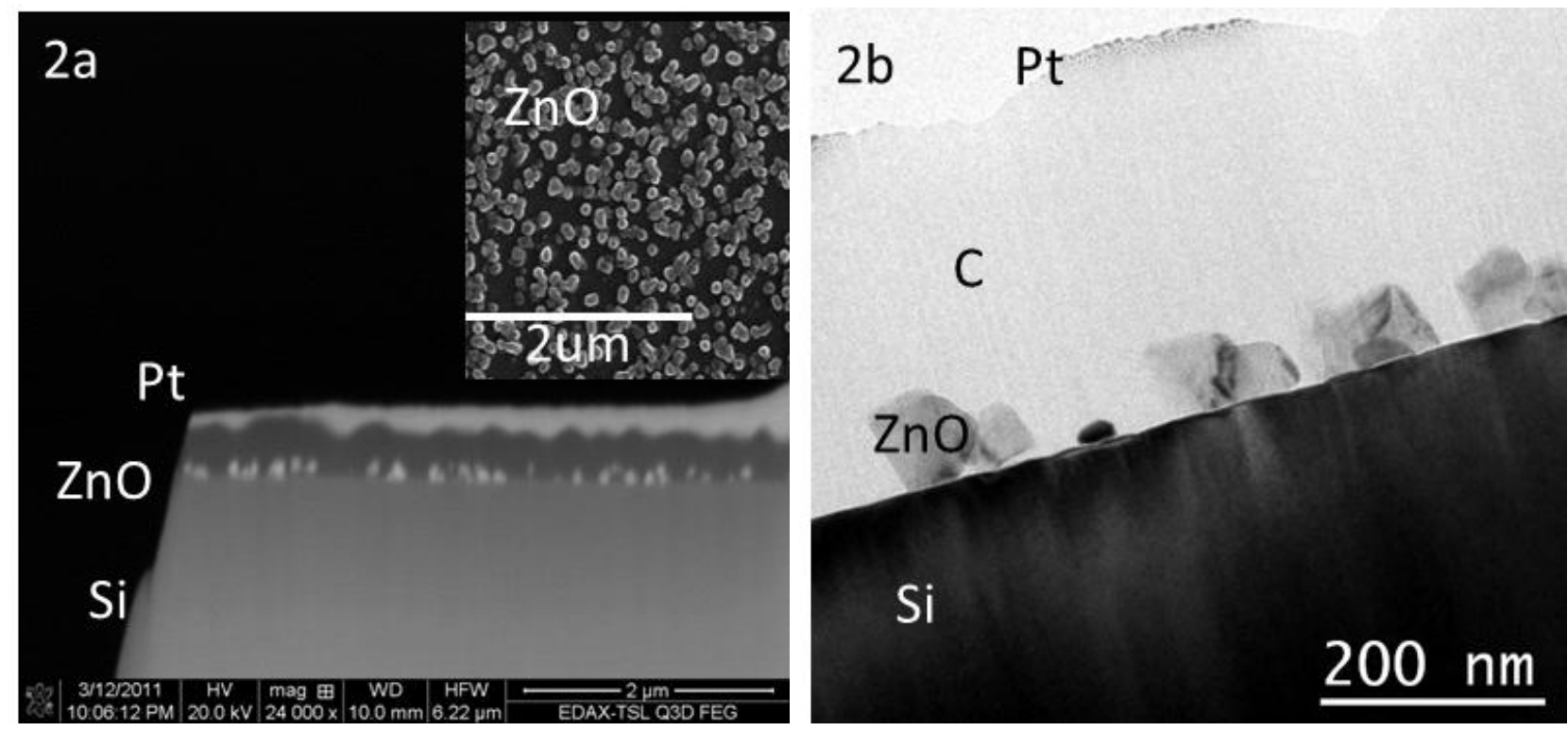

Fig. (2a) SEM image of the cross-sectional TEM specimen of $\mathrm{ZnO}$ nanocystals which are grown on Si substrate and used for resistive random access memory. The inset is a SEM image of $\mathrm{ZnO}$ nanocrystals before TEM specimen preparation. (2b) Final cross-sectional TEM specimen of ZnO nanocystals imaged in a TEM. 